\title{
Enhanced subsurface grain refinement during transient shear-based surface generation
}

\author{
Saurabh Basu, Zhiyu Wang, Ryan Liu, Christopher Saldana* \\ George W. Woodruff School of Mechanical Engineering, Georgia Institute of Technology, \\ 801 Ferst Dr, Atlanta, GA USA
}

\begin{abstract}
The present work combines quantitative orientation imaging microscopy and in situ digital image correlation to identify heterogeneities in the coupled mechanics and microstructure evolution occurring in the deformed subsurface during transient shear-based surface generation. Subsurface microstructure exhibited heterogeneities in terms of thickness of the ultrafine-grained layer and recrystallization fraction as a function of position along the surface wavelength. It was observed that subsurface microstructure evolution followed accelerated recrystallization kinetics due to strain path changes occurring in the subsurface during transient surface generation. The magnitudes of these strain path changes and pre-straining of the deformed subsurface were observed to correlate well with changes in the information entropy of the corresponding subsurface crystallographic textures. A phenomenological model for predicting the information entropy of the orientation distribution function based on strain path changes and strain history was formulated and validated for monotonic loading paths. The implications of this generalized framework for modeling and controlling subsurface microstructure in transient surface generation are briefly discussed.
\end{abstract}

*Corresponding author: christopher.saldana@me.gatech.edu 


\section{Introduction}

Functional response at interfaces of structural components is often strongly related to surficial topography and the corresponding microstructural state prevailing in the subsuface. For example, graded nano-scale microstructures characterized by grain size $\delta<0.5 \mu m$ on biomedical implant surfaces has been shown to enhance biocompatibility due to grain boundary effects on tissue integration [1]. Significant attention has been made to understand relationships between functional response and surficial microstructure/topography with the overarching goal of designing optimal engineered surfaces constituting a balance of both of these surface parameters. To this end, studies that delineate microstructural influences on response have been performed on topographically featureless surfaces [2]. Through these studies, it was shown that nanocrystalline, nanotwinned and ultra fine grain (UFG) microstructures exhibit better corrosion response [3], fatigue resistance [4] and thermal stability [5], respectively. Complimentary to these studies, single variable functional response of topographically feature rich surfaces have showed direct correlation between surface topography and performance measures such as bio-compatibility [6] and wear [7].

Research motivated towards enhancing functional response has been augmented with studies that aim to simultaneously control surface microstructure and topography. In this regard, thermal ablation using precisely controlled high energy laser or electron beams has been realized as a technique to imprint controlled topographical feature arrays $[8,9]$, as well as controlled microstructural characteristics [10]. While these techniques provide capability for high spatial resolution and precision, scalable implementation for controlled surface design is severely hampered by the high cost of capital equipment. In comparison, shear-based surface generation using transient material removal processes offers a scalable alternative for integrating sophisticated microstructural and topographical designs into fabricated components [ $[7,11]$. This process involves advancing a wedge shaped tool across a work surface in a periodic manner so to control the engagement and disengagement of the tool from the work surface. 
The resulting effect is a continuously varying surface topography which can be harnessed to yield a range of surface designs, including micro-scale ribs, fins and dimples $[7,11]$. While considerations pertaining to surface topography control have been well addressed, the integrated surface mechanics and microstructure consequences are not as well understood.

Microstructure evolution as a result of severe shear imposed in conventional surface generation involves interplay of dislocation storage and annihilation [12, 13], sometimes compounded by twinning [14] and often resulting in UFG and nanocrystalline microstructures. This evolution is determined by the imposed thermomechanical state $(\epsilon, \dot{\epsilon}, T)$ and corresponding process parameters including tool geometry, deformation rate and deformation volume $[15,16]$. Surface generation occurring in conventional material removal involves deformation by severe simple shear $\epsilon \gg 1$, resulting in spatially heterogeneous mechanics and microstructure evolution in the subsurface [17]. For transient material removal processes wherein operative process parameters are temporally-varying, spatial and temporal heterogeneity of mechanics and microstructure evolution are not as well understood. Macroscopic consequences of variation in process parameters include continuously varying forces [18] and specific energy of deformation [19]. However, implications of transient material removal on surficial microstructure characteristics are yet to be elucidated.

Of particular interest in transient material removal processes are effects of strain path changes imposed in the work surface by the periodic tool engagement/disengagement on subsurface microstructure evolution. Strain path changes cause altered grain refinement rates $\left|\frac{\partial \delta}{\partial \epsilon}\right|$, crystallographic textures, continuous dynamic recrystallization (CDRX) statistics, grain boundary orientations and grain morphologies. For instance, CDRX of FCC metals and alloys has been observed to occur in an accelerated manner as a result of shear imposed in different directions in equal channel angular pressing (ECAP) [20, 21, 22]. This resulted in larger grain refinement rates $\left|\frac{\partial \delta}{\partial \epsilon}\right|$ compared with monotonic deformation paths at similar imposed effective strains $\epsilon$. In the present study, evolution of microstructure and crystallographic texture during transient sur- 
face generation in OFHC Cu was investigated. From the present results, a phenomenological framework for predicting mechanics of microstructure evolution in transient surface deformation is discussed.

\section{Experimental methods}

A two-dimensional plane strain configuration shown in Fig. 1 was used to generate deformed surfaces in annealed OFHC Cu (grain size $\delta \sim 50 \mu m$ ). Control of the surface waveform was facilitated by advancing a hardened steel tool into the workpiece at a constant lateral velocity (X-direction) of $v_{x}=25 \mathrm{mms}^{-1}$ while si-

nusoidally modulating the tool vertical velocity (Y-direction) as $v_{y}(x)=0.314 v_{x} \cos \left(\frac{2 \pi x}{0.15}\right)$ $m m s^{-1}$, in the range $v_{y}=[-7.9,7.9] \mathrm{mms}^{-1}$. The resulting surface waveform was given by $a_{0}(x)=0.1+0.0075 \sin \left(\frac{2 \pi x}{0.15}\right)$ where all dimensions are in $\mathrm{mm}$. The tool had a nominal rake angle of $\alpha=0^{\circ}$, an included angle of $\beta=30^{\circ}$ and a clearance angle of $90^{\circ}-\beta=60^{\circ}$. The effective rake angle varied continuously along the surface wavelength due to the imposed sinusoidal vertical velocity. The effective rake angle is related to the ratio of the lateral and vertical velocities as $\alpha_{e f f}=-\tan ^{-1}\left(\frac{v_{y}}{v_{x}}\right)$ which, for the present conditions, varied over the range $\alpha_{\text {eff }}=\left[-17.44^{\circ}, 17.44^{\circ}\right]$. Additionally, several conventional surface generation experiments were also conducted with $v_{y}=0$ to enable in situ characterization of the deformation field. In situ characterization of the deformation zone during surface generation was facilitated using digital image correlation (DIC). DIC involves recording material flow in the deformation zone in a sequence of digital images and subsequently using an image correlation algorithm to characterize the resulting displacement fields. Prior utilization of this technique has proven to be valuable for characterizing deformation fields in a broad class of deformation configurations $[23,24,25,26]$. Quantitative orientation imaging microscopy (OIM) of the material state left in the wake of the tool was performed using electron back-scattered diffraction (EBSD). Sample preparation for OIM involved mechanical polishing to a mirror finish followed by ion polishing using a Hitachi 4000 plus ion polisher at $45 \mathrm{kV}$ and $20 \mathrm{~mA}$ for several minutes. A scan step size 
$<100 \mathrm{~nm}$ and electron beam diameter of $20 \mathrm{~nm}$ were utilized during OIM.

\section{Results}

Figure 2a shows OIM of the heterogeneous surface topography from which microstructure parameters pertaining to gradients in grain diameter $(\delta)$, recrystallization fraction $\left(f_{r x}\right)$, dislocation structure orientation and crystallographic texture were extracted. From the figure, a high concentration of UFGs was seen across the entire surface wavelength in the immediate subsurface, compared to microcrystalline grains observed at greater subsurface depths. Figure $2 \mathrm{~b}$ summarizes grain size measurements as a function of depth in the direction of the surface normal $(d)$ for two locations along the surface wavelength. From the figure, the gradient in grain size was steeper for $\frac{x}{\lambda} \sim 0.35$ in comparison to $\frac{x}{\lambda} \sim 0.6$, wherein grain size was defined as $\delta_{2^{\circ}}<0.5 \mu \mathrm{m}$ and $\delta_{2}$ 。 denotes grains comprised of boundaries having misorientation greater than $2^{\circ}$. The gradient in grain size $\left(\frac{\partial \delta_{2} \circ}{\partial d}\right)$ observed here is similar to that noted elsewhere for conventional material removal configurations [17]. Figure 3 shows higher resolution OIM of the subsurface microstructure field. The dashed white line in Fig. 3a demarcates the zone abutting the surface exhibiting presence of UFGs with $\delta_{15^{\circ}}<0.5 \mu \mathrm{m}$. From the micrograph, it is clear that this UFG layer thickness $d_{u f g}$ varied with respect to location along the surface wavelength. This variation, illustrated in Fig. 4, exhibits global and local maxima that are identified in the figure with arrows. The global maximum identified at $\frac{x}{\lambda} \sim 0.17$ corresponds to the location approximately halfway between points A and B in Fig. 3a. The smaller local maxima at $\frac{x}{\lambda} \sim 0.6$ corresponds to the zone within the dotted ellipse near apex $B$ in Fig. 3a. The UFG surficial zones exhibited pancake shaped grains with serrated boundaries impinging onto the surface, identified using white arrows in Fig. 3a and magnified in Fig. 3b. These features are indicative of geometric dynamic recrystallization $[27,28]$ wherein serrations on opposing sides of a grain impinge with imposition of incremental shear and result in formation of several offspring grains. 
Extent of recrystallization was quantified by determining recrystallization fraction, given as $f_{r x}=\frac{\ell_{H A G B}}{\ell_{L A G B}+\ell_{H A G B}}$, where $\ell_{L A G B}$ and $\ell_{H A G B}$ refer to length of low and high angle grain boundaries featuring disorientations of $2^{\circ}-15^{\circ}$ and $>15^{\circ}$, respectively. These fractions are shown in Fig. 3a and are correlated to the UFG layer thickness, wherein the largest recrystallization fraction $f_{r x}=0.67$ occurred where maximum UFG layer thickness was observed (e.g., $\frac{x}{\lambda} \sim 0.17$ ). Similarly, the smallest recrystallization fraction $f_{r x}=0.18$ was observed where the UFG layer thickness was also minimum (e.g., $\frac{x}{\lambda} \sim 0.75$ ). Interestingly, recrystallization fraction exhibited a local maximum $f_{r x}=0.58$ close to apex B (e.g., $\frac{x}{\lambda} \sim 0.6$ ), where a local maximum in UFG layer thickness was also seen. The heterogeneity in recrystallization fraction observed across the surface wavelength is indicative of transient effects in the mechanics of the surface generation.

Surficial crystallographic textures produced during transient surface generation were extracted from OIM data using the MATLAB based software MTEX [29] and subsequently reoriented in order to facilitate comparison with textures produced in conventional surface generation configurations [23]. This reorientation ensured that the representative direction of the tool was parallel to the horizontal (e.g., X direction) in the final ODFs. Herein, sections of the orientation distribution function (ODF) given by $\phi 2=\left\{0^{\circ}, 15^{\circ}, 30^{\circ}, 45^{\circ}, 60^{\circ}, 75^{\circ}, 90^{\circ}\right\}$ are elucidated in Fig. 5. These crystallographic textures reveal a near $\frac{2}{m}$ symmetry about the X-direction with high concentrations of orientations along fibers outlined using dashed lines. These fibers were coincident with those exhibited by conventional surface generation within experimental error and the intensities exhibited by the ODFs $(8,26)$ were also within a similar range [23]. However, qualitative examination of the present crystallographic textures suggests a degree of variation within the deformation mechanics during transient surface generation. For instance, crystallographic textures obtained from section $e$ in Fig. 5 exhibited a larger spread compared with that obtained from other sections.

Characterization of the deformation field by DIC was made to understand the deformation occurring in the surface generation process. Figure 6 shows results of in situ characterization of material flow during unit plane strain sur- 
face generation with $\alpha=-40^{\circ}$ and $a_{0}=75 \mu \mathrm{m}$. A representative image from the sequence capturing the state of material in the vicinity of the deformation zone is shown in Fig. 6a. The asperities present as bright/dark spots provided the inherent markers needed to map material flow fields during surface generation. The resulting velocity distribution is provided in Fig. 6b. Differentiation and temporal integration of velocity fields over material pathlines throughout the field provided evaluation of the imposed strain field as shown in Fig. 6c. The strain in the deformed subsurface can be modeled as: $\epsilon(d)=\epsilon_{0} \exp \left(-\kappa_{\alpha} \frac{d}{a_{0}}\right)$, where $a_{0}$ is the extent of the engagement of the tool with the workpiece and $\left\{\epsilon_{0}, \kappa_{\alpha}\right\}$ are fitting parameters dependent on $\alpha$ [17]. Variation in the effective strain field with respect to depth from the surface was fitted as $\epsilon_{d}=6.41 \exp \left(-115.08 \frac{d}{a_{0}}\right)$ with high $R^{2}>0.97$, shown in Fig. 6d. This variation was also characterized for $\alpha=-20^{\circ}$ as $\epsilon_{d}=5.01 \exp \left(-126.53 \frac{d}{a_{0}}\right)$ with high $R^{2}>0.97$. Variation in $\epsilon_{0}$ is largely influenced by parameter $\alpha$, wherein a more negative $\alpha$ results in an anticipated larger $\epsilon_{0}$. From the experimental conditions, this was characterized as $\epsilon_{0}=-0.0697 \alpha+3.6176$. Sim-

ilarly, variation in the parameter $\kappa$ with respect to $\alpha$ was characterized as $\kappa_{\alpha}=-0.6225 \alpha-139.98$. These experimental data are useful for understanding deformation levels in transient surface generation as described in the ensuing.

\section{Discussion}

The present results elucidate heterogeneities in subsurface microstructure and texture that are evolved during transient surface generation. The spatial and temporal heterogeneities in these microstructure-related characteristics are a consequence of variations present in the mechanics of the variable surface generation process. In the ensuing, this behavior is encapsulated within a framework for predicting microstructure fields resulting from the transient surface generation process, as well as for describing the role of strain path changes on the evolved subsurface microstructure. 


\subsection{Mechanics and microstructure evolution in transient surface generation}

Prior studies $[5,15,16,23,24]$ have shown that microstructure fields and crystallographic textures resulting from plastic deformation evolve as a consequence of the prevalent thermomechanics characterized in terms of $(\epsilon, \dot{\epsilon}, T)$. For subsurface deformation occurring during surface generation, attempts have been made to describe this triad as a function of deployed processing parameters in terms of tool rake angle $\alpha$ and deformation rate $v$. Fidelity of frameworks in modeling microstructural consequences resulting from the deformation imposed during surface generation have been demonstrated across a broad class of materials and thermomechanical subspace [15].

The state of the deformed surface in terms of the imposed effective strains in conventional surface generation (i.e., $\left.v_{y}=0\right)$ is given by $\epsilon_{d}=\epsilon_{0} \exp \left(-\kappa_{\alpha} \frac{d}{a_{0}}\right)$, where $\kappa_{\alpha}$ is a parameter dependent solely on $\alpha$ and $\epsilon_{0}$ is the surface effective strain correlated with $\alpha$ through effective strain imposed in the removal volume (chip) [30]. As $\alpha$ is constant in conventional surface generation, the subsurface strain field is a steady-state but spatially heterogeneous deformation field. This is not the case for a transient surface generation process as the effective rake angle takes on a range of values depending on the instantaneous position along the surface waveform. For the experimental conditions explored in the present study, this resulted in $\alpha_{e f f} \in\left[-17.44^{\circ}, 17.44^{\circ}\right]$ during the surface generation process. Further, the DIC measurements yielded $\epsilon_{0}=-0.0697 \alpha+3.6176$ and $\kappa_{\alpha}=-0.6225 \alpha-139.98$. The resulting subsurface deformation field is shown in Fig. 7a. From the figure, it is clear that the strain field is heterogeneous along the surface wavelength wherein regions of high strain correspond to the rising surface edge (i.e., $\left.\alpha_{e f f}<0\right)$ and regions of low strain correspond to the falling surface edge (i.e., $\alpha_{e f f}>0$ ). Further, asymmetry in the strain field is clearly evident due to the transient nature of the subsurface deformation field along the surface wavelength.

Comparison of the subsurface effective strain field with the microstructure of the deformed subsurface suggests activation of continuous recrystallization with the imposition of large plastic strain in the subsurface. Imposition of 
increasing levels of strain generally results in the formation of incidental dislocation boundaries (IDBs) and geometrically necessary boundaries (GNBs). The former results from probabilistic events constituting the statistical interlocking of dislocations whereas the latter results from geometrical necessity in order to maintain material continuity [12]. Progressive imposition of strains causes misorientation featured by IDBs to increase, eventually resulting in their progression to GNBs. The spacing between these boundaries decreases with imposition of larger strains, resulting in microstructure refinement. In this regard, grain refinement within the subsurface during transient surface generation is schematically illustrated in Fig. 7b. The microstructure field quantified in Fig. $2 \mathrm{~b}$ along directions $i$, ii depicted in Fig. 2a exhibited an increase in grain size $\delta_{2}$ 。 harmoniously with respect to decreasing strains along these directions. The microstructure fields also exhibited a similar evolution in dislocation boundaries and their consolidation to form new grains as depicted in insets i and ii in Fig. $7 \mathrm{~b}$, wherein inset ii in the near-surface exhibits a more refined grain structure due to higher imposed strains and that of inset $i$ in the far field exhibits less refinement due to lower imposed strains. This mechanism of microstructure evolution is often accompanied by shear-induced pancaking of grains surrounded by high angle grain boundaries (HAGBs) featuring misorientation $\theta>15^{\circ}$. High aspect ratio pancake-shaped grains tend to develop serrations on their boundaries [27] that eventually subdivide a single grain to several off-spring grains upon pinching with their counterparts in the facing boundary. Herein, instances of grain pancaking and serration were observed near apex B in the surface wavelength, locally identified using the white arrow in the segment $A B$ in Fig. 3a and Fig. 3b. Finally, the location exhibiting minimum effective strains in the strain field depicted in Fig. 7a using the asterisk corresponds to the zone that exhibits a near-pristine equi-axed grain structure.

Progression in strain induced recrystallization of new grains was quantified by extracting the parameter $f_{r x}=\frac{\ell_{H A G B}}{\ell_{L A G B}+\ell_{H A G B}}$ close to the surface using results obtained from OIM. This characterization is depicted as numbers over the topography in Fig. 3a. This parameter, also known as the high angle grain 
boundary fraction assumes a value close to 1 after recrystallization of new grains, these being essentially defect free upon their genesis and lacking low angle grain boundaries $[27,28]$. From the present results, $f_{r x}$ exhibited a cyclical variation with respect to location along the surface wavelength. The maximum value of $f_{r x}=0.67$ coincided directly with the location $\frac{x}{\lambda}=0.17$ that exhibited maximum surficial strain $\epsilon \sim 4.8$ as well as global maximum in UFG layer thickness $d_{u f g} \sim 5 \mu m$. Similarly, the location $\frac{x}{\lambda} \sim 0.75$ with minimum $f_{r x}=0.18$ coincided with lowest surficial strain $\epsilon \sim 2.4$ and smallest $d_{u f g} \sim 0.5 \mu m$. Further positive correlation between $f_{r x}, \epsilon$ and $d_{u f g}$ was seen at other positions along the surface wavelength of $\frac{x}{\lambda}=\{0.28,0.40\}$ whereby surface strains of $\epsilon=\{4.8,4.3\}$ yielded $f_{r x}=\{0.60,0.47\}$, along with a decrease in the UFG layer thickness $d_{u f g}=\{3 \mu m, 1.1 \mu m\}$. Closer inspection of the coupled variation in $f_{r x}$ and $\epsilon$ in the subsurface revealed evolution that cannot be explained by a monotonic relationship between these two parameters. For example, a negative correlation between these parameters was observed for $\frac{x}{\lambda}=\{0.40,0.51\}$, which corresponded to $\epsilon=\{4.3,3.6\}$ and $f_{r x}=\{0.47,0.58\}$. This feature was reflected onto the observed variation of parameter $d_{u f g}$ which also exhibited an increase from $1 \mu m$ to $2 \mu m$ across these locations, despite concomitantly decreasing strains. This non-monotonic response is considered in the ensuing.

\subsection{Accelerated microstructure evolution in transient surface generation}

Recent investigations have observed non-monotonic relationships between $f_{r x}$ and $\epsilon$ caused by presence of strain path changes due to concomitant rises in local plastic spin rates $[21,24]$. The latter is caused by activation of additional slip systems in the presence of strain path changes, essentially manifesting a faster decline in grain size with respect to imposed effective strains as depicted in Fig. $7 \mathrm{~b}$. The influence of strain path changes in accelerating rates of microstructure evolution with respect to strains imposed have also been recognized in a broader class of materials. For instance, the rate of grain refinement characterized as $\left|\frac{\partial \delta}{\partial \epsilon}\right|$ was seen to increase in the $B$ route of ECAP in commercially pure $\mathrm{Al}$, this featuring larger strain path changes with respect to the other routes relevant 
to the same deformation platform [22]. Mechanistic models have been proposed that simulate the influence of strain path changes on the resulting mechanical behavior and concomitant microstructure evolution of the specimen undergoing deformation [20]. However, single valued parameters that aim to encapsulate the effect of strain path changes on the resulting material response have not been well studied despite these strongly affecting the resulting functional response of the process. Further, the $f_{r x}$ parameter is determined purely from the grain boundary length statistics of a polycrystal and does not encapsulate orientation-related data, the latter being crucial in a comprehensive description of microstructure.

The information entropy of the ODF offers a potential alternative parameter for understanding microstructure evolution in the context of strain path changes. Information entropy, also known as the Shannon's entropy, is defined as $H=-\sum_{i}^{n} p_{i} \log p_{i}$ and characterizes the entropy of information contained in a random variable $X$ that can exhibit $n$ values with respective probabilities given by $p_{i}$. In the present context, $\mathrm{X}$ is related to the ODF of a microstructure wherein randomly sampling from the microstructural volume could result in a grain with an orientation $g$ with probability $p=f(g)$. Here, $f(g)$ is defined as the volume fraction of material in the specimen that features orientation $g$. Probabilistic sampling from the ODF has been demonstrated elsewhere in calculating expected values of physical properties such as Young's modulus given a specific crystallographic texture [31, 32]. Further, maximization of information entropy of orientation distributions (OD) has also been used to directly calculate ODs from pole figures [33]. In this regard, the information entropy is calculated using orientation data that in itself does not take into account the concentration of atomic defects in the crystals within the polycrystalline volume [34]. The latter is directly related to the free energy of the system, this related to thermodynamical entropy as opposed to information entropy [35][36].

Dependence of ODF information entropy on plastic deformation arises as different deformation modes result in unique crystallographic textures [23, 24, $32,37]$. For instance, simple shear results in an ODF concentrated in char- 
acteristic fibers viz. $f 1, f 2, f 3$, whereas rolling deformation results in the $\alpha$ and $\beta$ fiber. These crystallographic textures saturate with increasing strain in their defining characteristics including strengths and fiber locations. Herein it is noted that presence of specific fibers in crystallographic textures increases the probability that a randomly sampled grain orientation belongs to that fiber, thereby decreasing the information entropy. In this regard, the entropy of the respective ODFs also saturates to a minimum with increasing strain, this governed by the prevalent deformation mode and its characteristic fibers. This ansatz is demonstrated in Fig. 8a that shows decreasing information entropy with increasing strain for deformation by compression, simple shear and rolling. These ODFs were calculated using the calibrated VPSC framework described in Refs. $[23,24,38]$ with an effective strain rate $\dot{\epsilon} \sim 1 s^{-1}$. Further, it is noted that activation of multiple deformation modes or strain path changes will result in evolution of new fibers in the ODFs, increasing information entropy until sufficient strains are imposed. This behavior is demonstrated in Fig. 8b, where the influence of compressive deformation on pre-rolled microstructures featuring rolling fibers in their ODFs is shown. The compressive deformation was imposed after pre-strains of $\epsilon \sim\{0.5,1\}$ in the transverse direction. From the figure, it is clear that the strain path change acts to increase information entropy of the ODF, which then saturates at higher effective strains. From the figure, strain path changes imposed after a pre-strain of $\epsilon \sim 1$ resulted in a significantly larger change in parameter $H$, compared with those at smaller strain $\epsilon=0.5$. This change was characterized by a + ve $\frac{\partial^{2} H}{\partial \epsilon^{2}}$ for both levels of pre-strain. Additionally, at larger pre-strains, +ve $\frac{\partial H}{\partial \epsilon}$ was also seen.

For transient surface generation, the information entropy of the ODF is dependent on the strain path change and the surface pre-strain. In this transient process, strain path changes are manifested due to locally varying shear directions occurring along the surface wavelength. The direction of shear in the deformed surface has been observed to lie near parallel to the surface tangent [23]. In this regard, the direction of the eigenvector of the surficial deformation tensor also lies in a near parallel orientation relative to the surface, these de- 
picted using arrows in Fig. 7a for our experimental platform. The direction of the eigenvector was characterized as $\tan \theta_{\tau}=\frac{\partial a_{0}(x)}{\partial x}$ and the resulting imposed strain path changes quantified as $\frac{\partial \theta_{\tau}}{\partial x}=\left(1+\left(\frac{\partial a_{0}(x)}{\partial x}\right)^{2}\right)^{-1}$. Further, the influence of these path changes on information entropy of the ODF are proportional to the imposed pre-strain as larger pre-strains will act to consolidate crystallographic texture within well defined fibers featuring narrow spreads, whereby small subsequent changes in strain path will result in discernible deviations and instabilities in the developed texture [39]. In surface generation, the origin of the pre-strain is in the subsurface deformation zone extending ahead of the tool during its advance, also depicted using DIC in Fig. 6c. While in conventional surface generation this pre-strain is spatially uniform in the direction of the surface tangent, this is not the case for transient surface generation due to a continuously varying surface geometry. In this regard, the pre-strain at location $x$ originates from deformation imposed by the approaching tool while it is at $x^{-}$ and the lag can be characterized as a phase angle offset $\gamma=\frac{x^{-}-x}{\lambda}$ which yields a pre-strain influence given by $\epsilon_{\gamma}^{\nu}$, where $\nu>0$ and $\gamma<0$. At greater imposed final strains $\epsilon$ during surface generation, the effect of these pre-strains is decreased, consistent with the observed behavior for other deformation modes in Fig. 8. These combined effects in terms of the proportionality of the information entropy of the ODF with strain path changes and the imposed pre-strain, as well as the dominating influence of the imposed final strain, can be encapsulated as $H \propto\left(\frac{\partial \theta_{\tau}}{\partial x}\right)^{\psi} \frac{\epsilon_{\gamma}^{\nu}}{\epsilon^{\xi}}$, where $\xi, \psi, \nu>0$ and $\gamma<0$.

The numerical information entropy of the ODFs were extracted from the experimental texture measurements as $H=-\Sigma_{\Omega} f(g) \log f(g)$. Here, $\Omega$ refers to the orientation space and $\frac{f(g)}{8 \pi^{2}} d g \equiv \frac{f(\phi 1, \Phi, \phi 2)}{8 \pi^{2}} d g$ refers to the probability that a randomly chosen point within the volume of the polycyrstal features an orientation given by $g \equiv(\phi 1, \Phi, \phi 2)$. The resulting entropy exhibited cyclical behavior with respect to position along the surface wavelength, this featuring a bowl shaped variation as $H \sim\{3.775,3.191,3.244,3.261,3.633\}$ at positions of $\frac{x}{\lambda} \sim\{0,0.25,0.5,0.75,1\}$ and surface strains $\epsilon \sim\{3.6,4.8,3.6,2.4,3.6\}$, respec- 
tively. Inspection of the information entropy parameters $H$ extracted from OIM of microstructures created during transient surface generation showed that this parameterization can be encapsulated as: $H=3.49\left(\frac{\partial \theta_{\tau}}{\partial x} \frac{\epsilon_{-0.23}}{\epsilon}\right)^{0.90}$ with $R^{2} \sim 0.97$. Herein, the parameter $\gamma=-0.23 \mathrm{rad}$ corresponds to a physical distance $x-x^{-}=34 \mu \mathrm{m}$, within the same order of magnitude as the expected physical dimensions of the subsurface deformation zone, this being the origin of the pre-strain during transient surface generation. The fitted expression supports the expected relationships between the individual parameters and the information entropy of the ODF. For instance, a + ve parameter $\nu$ is consistent with the earlier observation that ODF information entropy is directly correlated with pre-strain imposed prior to the strain path change, as in the simulated deformation of Fig. 8b. Crystal plasticity origins of this phenomenon can be found in the latent hardening of crystals within the polycrystal. Monotonic strain paths are often dominantly accommodated by dislocation slip activity in less than five slip systems up to moderate strains, contrary to the Taylor criterion [37]. However, due to latent hardening in FCC polycrystals, slip activity in system $\alpha$ results in simultaneous hardening of other systems $\beta$, these coupled using the latent hardening coefficient matrix denoted $h_{\alpha \beta}$ [40]. In this regard, a larger pre-strain results in larger latent hardening, which in turn manifests in the formulation comprising the power-law relationship with the pre-strain.

The functional form $H=\mathcal{C}_{1}\left(\frac{\partial \theta_{\tau}}{\partial x}\right)^{\psi} \frac{\epsilon_{\gamma}^{\nu}}{\epsilon}$, which aims to encapsulate the variation of information entropy during transient surface generation, was validated with results obtained from numerical experiments. In this regard, it was realized that step changes in the strain path, such as those illustrated in Fig. 8b must incorporate a constant strain path change factor as $H \propto \mathcal{C}_{2} \frac{\epsilon_{r}^{\nu}}{\epsilon_{c}^{\xi}}$, in comparison with transient shear based surface generation. Here, $\epsilon_{r}$ and $\epsilon_{c}$ correspond to rolling pre-strain and compression post-strain, respectively. It was seen that this formulation successfully encapsulated variation in numerically calculated entropies during step strain path changes as $H=3.83\left(\frac{\epsilon_{r}}{\epsilon_{c}}\right)^{0.039}$ with $R^{2} \sim 0.93$ and $R^{2} \sim 0.74$, for $\epsilon_{r}=0.5$ and 1.0, respectively. This variation is depicted for $\epsilon_{r}=0.5$ and 1.0 in Fig. $8 \mathrm{~b}$ and for a broader range of rolling pre-strains, fol- 
lowed by compressive post-strains in Fig. 9. It is noted that this formulation does not take into account the crystal plasticity phenomena during the onset of the strain path change that manifest the non-monotonic derivatives $\frac{\partial H}{\partial \epsilon}$ and $\frac{\partial^{2} H}{\partial \epsilon^{2}}$. Further, imposition of a step strain-path changes result in significantly slower evolution in $H$ with respect to cumulative strains, as characterized using $\xi=0.039$, compared with $\xi=0.9$ for transient surface generation, this observation replicable numerically. While crystal plasticity origins of this phenomenon are not delineated here, the present characterization of $H$ as a function of the imposed deformation histories is useful for modeling controllability of surficial microstructures for severe shear based material processing platforms. These generalized frameworks are envisioned to provide data for controlling functional properties of fabricated components, these being closely linked to the underlying microstructural characteristics.

\section{Conclusion}

Evolution of subsurface microstructure during transient surface generation in OFHC $\mathrm{Cu}$ was investigated using EBSD-based OIM characterization. The deformed subsurface was found to consist of a graded ultrafine-grained microstructure. In this regard, the thickness of this ultrafine-grained layer was observed to vary as a function of position along the surface wavelength. Analysis of recrystallization fraction in the microstructure of the deformed surface showed evidence of accelerated microstructure evolution and refinement during the imposed surficial deformation. Sources of this accelerated refinement were found in strain path changes induced by the continuously varying thermomechanical conditions present in transient surface generation. The influence of these strain path changes on microstructure evolution was quantified by consideration of the information entropy of crystallographic textures in the deformed surface. From these observations, it was seen that larger pre-strains imposed prior to these strain path changes resulted in larger final entropy. The dependence of information entropy on strain path changes and the imposed pre-strain was fur- 
ther found to be consistent with simulated evolution of information entropy of ODFs in different monotonic and non-monotonic deformation modes using the visco-plastic self-consistent framework.

\section{Acknowledgments}

The work at Georgia Tech was supported by NSF grant CMMI1254818 and the US Army Research Office (Agreement No. W911NF-14-1-0566).

\section{References}

[1] J.-W. Park, Y.-J. Kim, C. H. Park, D.-H. Lee, Y. G. Ko, J.-H. Jang, C. S. Lee, Enhanced osteoblast response to an equal channel angular pressing-processed pure titanium substrate with microrough surface topography, Acta Biomaterialia 5 (8) (2009) 3272 - 3280. doi:http://dx.doi.org/10.1016/j.actbio.2009.04.038.

URL http://www.sciencedirect.com/science/article/pii/ S1742706109002025

[2] C. N. Elias, M. A. Meyers, R. Z. Valiev, S. N. Monteiro, Ultrafine grained titanium for biomedical applications: An overview of performance, Journal of Materials Research and Technology 2 (4) (2013) 340 - 350. doi:http://dx.doi.org/10.1016/j.jmrt.2013.07.003.

URL http://www.sciencedirect.com/science/article/pii/ S2238785413000902

[3] Z. Pu, S. Yang, G.-L. Song, O. D. Jr., D. Puleo, I. Jawahir, Ultrafinegrained surface layer on mg-al-zn alloy produced by cryogenic burnishing for enhanced corrosion resistance, Scripta Materialia 65 (6) (2011) 520 523. doi:http://dx.doi.org/10.1016/j.scriptamat.2011.06.013.

URL

http://www.sciencedirect.com/science/article/pii/ S1359646211003411 
[4] T. Roland, D. Retraint, K. Lu, J. Lu, Fatigue life improvement through surface nanostructuring of stainless steel by means of surface mechanical attrition treatment, Scripta Materialia 54 (11) (2006) 1949 - 1954. doi:http://dx.doi.org/10.1016/j.scriptamat.2006.01.049.

URL http://www.sciencedirect.com/science/article/pii/ S135964620600131X

[5] C. Saldana, A. King, S. Chandrasekar, Thermal stability and strength of deformation microstructures in pure copper, Acta Materialia 60 (10) (2012) 4107 - 4116. doi :http://dx.doi.org/10.1016/j.actamat. 2012. 04.022 .

URL http://www.sciencedirect.com/science/article/pii/ S1359645412002789

[6] D. D. Deligianni, N. D. Katsala, P. G. Koutsoukos, Y. F. Missirlis, Effect of surface roughness of hydroxyapatite on human bone marrow cell adhesion, proliferation, differentiation and detachment strength, Biomaterials 22 (1) (2000) 87 - 96. doi:http://dx.doi.org/10.1016/S0142-9612(00)00174-5.

URL http://www.sciencedirect.com/science/article/pii/ S0142961200001745

[7] R. Gandhi, D. Sebastian, S. Basu, J. Mann, P. Iglesias, C. Saldana, Surfaces by vibration/modulation-assisted texturing for tribological applications, The International Journal of Advanced Manufacturing Technology (2015) 1-12doi:10.1007/s00170-015-7968-3.

URL http://dx.doi.org/10.1007/s00170-015-7968-3

[8] A. Ben-Yakar, R. L. Byer, A. Harkin, J. Ashmore, H. A. Stone, M. Shen, E. Mazur, Morphology of femtosecond-laser-ablated borosilicate glass surfaces, Applied Physics Letters 83 (15) (2003) 3030-3032. doi:http://dx.doi.org/10.1063/1.1619560. 
URL http://scitation.aip.org/content/aip/journal/apl/83/15/ $10.1063 / 1.1619560$

[9] D. Gilbert, M. Stoesslein, D. Axinte, P. Butler-Smith, J. Kell, A time based method for predicting the workpiece surface microtopography under pulsed laser ablation, Journal of Materials Processing Technology 214 (12) (2014) 3077 - 3088. doi:http: //dx.doi.org/10.1016/j.jmatprotec. 2014.07.008.

URL http://www.sciencedirect.com/science/article/pii/ S0924013614002672

[10] A. Kumar, T. M. Pollock, Mapping of femtosecond laser-induced collateral damage by electron backscatter diffraction, Journal of Applied Physics 110 (8). doi:http://dx.doi.org/10.1063/1.3653839.

URL http://scitation.aip.org/content/aip/journal/jap/110/8/ $10.1063 / 1.3653839$

[11] J. Mann, C. Saldana, W. Moscoso, Control systems and methods for machining operations, uS Patent 8,694,133 (Apr. 8 2014).

URL https://www.google.com/patents/US8694133

[12] D. Hughes, N. Hansen, Microstructure and strength of nickel at large strains, Acta Materialia 48 (11) (2000) 2985 - 3004. doi:http://dx.doi.org/10.1016/S1359-6454(00)00082-3.

URL http://www.sciencedirect.com/science/article/pii/ S1359645400000823

[13] E. Nes, Modelling of work hardening and stress saturation in $\{$ FCC $\}$ metals, Progress in Materials Science 41 (3) (1997) 129 - 193. doi:http://dx.doi.org/10.1016/S0079-6425(97)00032-7. URL http://www.sciencedirect.com/science/article/pii/ S0079642597000327

[14] J. Cai, S. Shekhar, J. Wang, M. R. Shankar, Nanotwinned microstructures from low stacking fault energy brass by high-rate se- 
vere plastic deformation, Scripta Materialia 60 (8) (2009) 599 - 602. doi:http://dx.doi.org/10.1016/j.scriptamat.2008.12.024.

URL http://www.sciencedirect.com/science/article/pii/ S1359646208008683

[15] S. Shekhar, S. Abolghasem, S. Basu, J. Cai, M. R. Shankar, Effect of severe plastic deformation in machining elucidated via rate-strain-microstructure mappings, Journal of Manufacturing Science and Engineering 134 (3) (2012) 031008-031008.

URL http://dx.doi.org/10.1115/1.4006549

[16] S. Abolghasem, S. Basu, S. Shekhar, J. Cai, M. Shankar, Mapping subgrain sizes resulting from severe simple shear deformation, Acta Materialia 60 (1) (2012) 376 - 386. doi:http: //dx.doi.org/10.1016/j.actamat.2011.09.055.

URL http://www.sciencedirect.com/science/article/pii/ S1359645411007026

[17] Y. Guo, C. Saldana, W. D. Compton, S. Chandrasekar, Controlling deformation and microstructure on machined surfaces, Acta Materialia 59 (11) (2011) 4538 - 4547. doi:http://dx.doi.org/10.1016/j.actamat. 2011. 03.076 .

URL http://www.sciencedirect.com/science/article/pii/ S1359645411002412

[18] H. T. III, W. Compton, S. Chandrasekar, A study of the influence of superimposed low-frequency modulation on the drilling process, Precision Engineering 22 (1) (1998) 1 - 9 . doi:http: //dx.doi.org/10.1016/S0141-6359(97)00085-8.

URL http://www.sciencedirect.com/science/article/pii/ S0141635997000858

[19] H. Yeung, N. K. Sundaram, J. B. Mann, W. D. Compton, S. Chandrasekar, Energy dissipation in modulation assisted machining, Inter- 
national Journal of Machine Tools and Manufacture 74 (2013) 41 - 49. doi:http://dx.doi.org/10.1016/j.ijmachtools.2013.07.007. URL http://www.sciencedirect.com/science/article/pii/ S0890695513001247

[20] I. J. Beyerlein, C. N. Tomé, Modeling transients in the mechanical response of copper due to strain path changes, International Journal of Plasticity 23 (4) (2007) 640 - 664. doi:http: //dx.doi.org/10.1016/j.ijplas.2006.08.001.

URL http://www.sciencedirect.com/science/article/pii/ S0749641906001197

[21] C. Gu, L. Tóth, M. Arzaghi, C. Davies, Effect of strain path on grain refinement in severely plastically deformed copper, Scripta Materialia 64 (3) (2011) 284 - 287. doi:http://dx.doi.org/10.1016/j.scriptamat. 2010.10 .002 .

URL http://www.sciencedirect.com/science/article/pii/ $\mathrm{S} 1359646210006834$

[22] Y. Iwahashi, Z. Horita, M. Nemoto, T. G. Langdon, The process of grain refinement in equal-channel angular pressing, Acta Materialia 46 (9) (1998) 3317 - 3331. doi:http://dx.doi.org/10.1016/S1359-6454(97) 00494-1.

URL http://www.sciencedirect.com/science/article/pii/ S1359645497004941

[23] S. Basu, M. Ravi Shankar, Crystallographic textures resulting from severe shear deformation in machining, Metallurgical and Materials Transactions A 46 (2) (2015) 801-812. doi:10.1007/s11661-014-2672-8.

URL http://dx.doi.org/10.1007/s11661-014-2672-8

[24] S. Basu, Z. Wang, C. Saldana, Anomalous evolution of microstructure and crystallographic texture during indentation, Acta Materialia 105 (2016) 25 - 34. doi:http://dx.doi.org/10.1016/j. actamat.2015.12.028. 
URL

http://www.sciencedirect.com/science/article/pii/ S1359645415301373

[25] F. Du, S. Yadav, C. Moreno, T. G. Murthy, C. Saldana, Incipient straining in severe plastic deformation methods, Journal of Materials Research 29 (2014) 718-728. doi:10.1557/jmr.2014.26.

URL http://journals.cambridge.org/article_S0884291414000260

[26] T. G. Murthy, C. Huang, S. Chandrasekar, Characterization of deformation field in plane-strain indentation of metals, Journal of Physics D: Applied Physics 41 (7) (2008) 074026.

URL http://stacks.iop.org/0022-3727/41/i=7/a=074026

[27] S. Abolghasem, S. Basu, M. R. Shankar, Quantifying the progression of dynamic recrystallization in severe shear deformation at high strain rates, Journal of Materials Research 28 (2013) 2056-2069. doi:10.1557/jmr. 2013.201.

URL http://journals.cambridge.org/article_S088429141300201X

[28] F. Humphreys, M. Hatherly, Recrystallization and Related Annealing Phenomena, 2nd Edition, Elsevier, 2004.

[29] R. Hielscher, H. Schaeben, A novel pole figure inversion method: specification of the MTEX algorithm, Journal of Applied Crystallography 41 (6) (2008) 1024-1037. doi:10.1107/S0021889808030112.

URL http://dx.doi.org/10.1107/S0021889808030112

[30] P. Guo, K. F. Ehmann, An analysis of the surface generation mechanics of the elliptical vibration texturing process, International Journal of Machine Tools and Manufacture 64 (2013) 85 - 95. doi:http://dx.doi.org/10.1016/j.ijmachtools.2012.08.003.

URL http://www.sciencedirect.com/science/article/pii/ S0890695512001599 
[31] R. Liu, A. Kumar, Z. Chen, A. Agrawal, V. Sundararaghavan, A. Choudhary, A predictive machine learning approach for microstructure optimization and materials design, Scientific Reports 5 (2015) 11551 EP.

URL http://dx.doi.org/10.1038/srep11551

[32] U. F. Kocks, C. N. Tomé, H.-R. Wenk, Texture and anisotropy: preferred orientations in polycrystals and their effect on materials properties, Cambridge university press, 2000.

[33] H. Schaeben, Entropy optimization in quantitative texture analysis. ii. application to poletoorientation density inversion, Journal of Applied Physics 69 (3) (1991) 1320-1329. doi:http://dx.doi.org/10.1063/1.347267.

URL http://scitation.aip.org/content/aip/journal/jap/69/3/10. $1063 / 1.347267$

[34] F. Humphreys, Characterisation of fine-scale microstructures by electron backscatter diffraction (ebsd), Scripta Materialia 51 (8) (2004) 771 - 776, viewpoint set no. 35. Metals and alloys with a structural scale from the micrometer to the atomic dimensions. doi:http://dx.doi.org/10.1016/j.scriptamat.2004.05.016.

URL http://www.sciencedirect.com/science/article/pii/ S1359646204002854

[35] G. Libowitz, Defect equilibria in solids, in: N. Hannay (Ed.), The Chemical Structure of Solids, Vol. 1 of Treatise on Solid State Chemistry, Springer US, 1921, pp. 335-385. doi:10.1007/978-1-4684-2661-8_6.

URL http://dx.doi.org/10.1007/978-1-4684-2661-8_6

[36] M. Nosonovsky, S. K. Esche, A paradox of decreasing entropy in multiscale monte carlo grain growth simulations, Entropy 10 (2) (2008) 49. doi: 10.3390/entropy-e10020049.

URL http://www.mdpi.com/1099-4300/10/2/49

[37] W. F. Hosford, The mechanics of crystals and textured polycrystals, New York : Oxford University Press, 1993., 1993. 
[38] S. Li, I. J. Beyerlein, D. J. Alexander, S. C. Vogel, Texture evolution during multi-pass equal channel angular extrusion of copper: Neutron diffraction characterization and polycrystal modeling, Acta Materialia 53 (7) (2005) 2111 - 2125. doi:http: //dx.doi.org/10.1016/j.actamat.2005.01.023.

URL http://www.sciencedirect.com/science/article/pii/ S1359645405000418

[39] Y. Zhou, L. Tth, K. Neale, On the stability of the ideal orientations of rolling textures for f.c.c. polycrystals, Acta Metallurgica et Materialia 40 (11) (1992) 3179 - 3193. doi:http: //dx.doi.org/10.1016/0956-7151(92)90481-S.

URL http://www.sciencedirect.com/science/article/pii/ $095671519290481 \mathrm{~S}$

[40] F. Roters, P. Eisenlohr, L. Hantcherli, D. Tjahjanto, T. Bieler, D. Raabe, Overview of constitutive laws, kinematics, homogenization and multiscale methods in crystal plasticity finite-element modeling: Theory, experiments, applications, Acta Materialia 58 (4) (2010) 1152 - 1211. doi:http://dx.doi.org/10.1016/j.actamat.2009.10.058.

URL http://www.sciencedirect.com/science/article/pii/ S1359645409007617

\section{Figures}




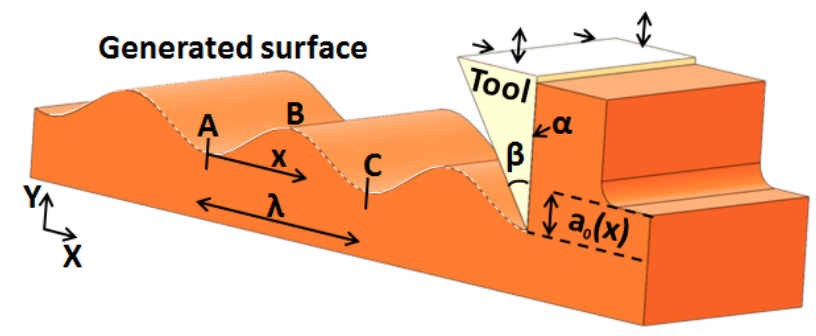

Figure 1: Experimental configuration for transient surface generation.

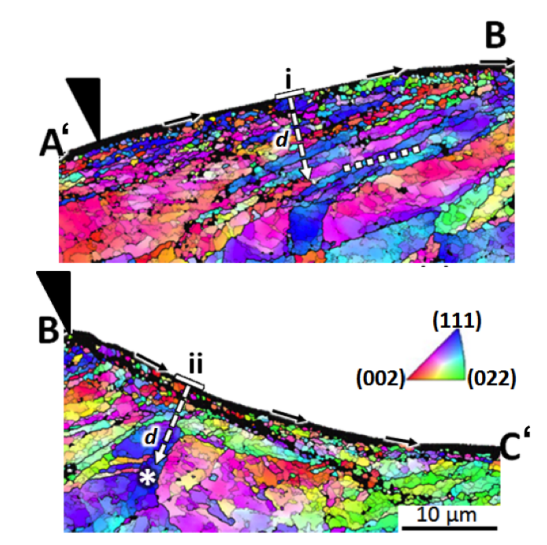

(a)

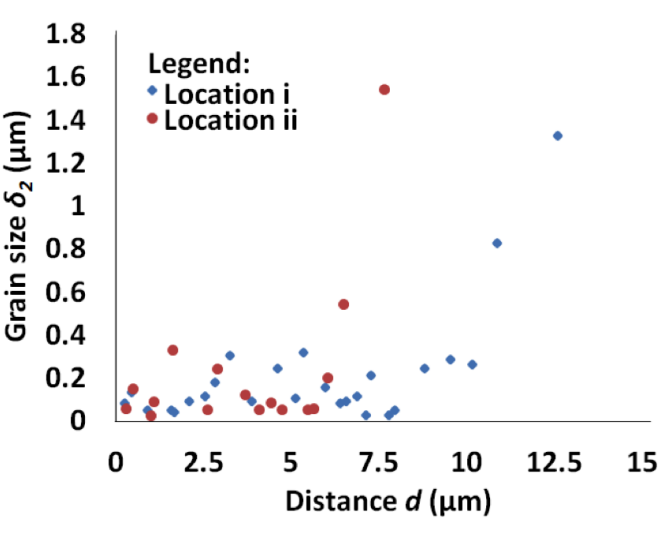

(b)

Figure 2: (a) OIM of subsurface microstructure characterized using EBSD for $\mathrm{A}^{`} \mathrm{~B}$ with $\frac{x}{\lambda}=[0.2,0.5]$ and $\mathrm{BC}^{\prime}$ with $\frac{x}{\lambda}=[0.5,0.8]$. (b) Gradient in grain size $\delta_{2} \circ$ with respect to increasing distance $d$ along lines marked using $i$ and $i i$ in Fig. 2(a). 

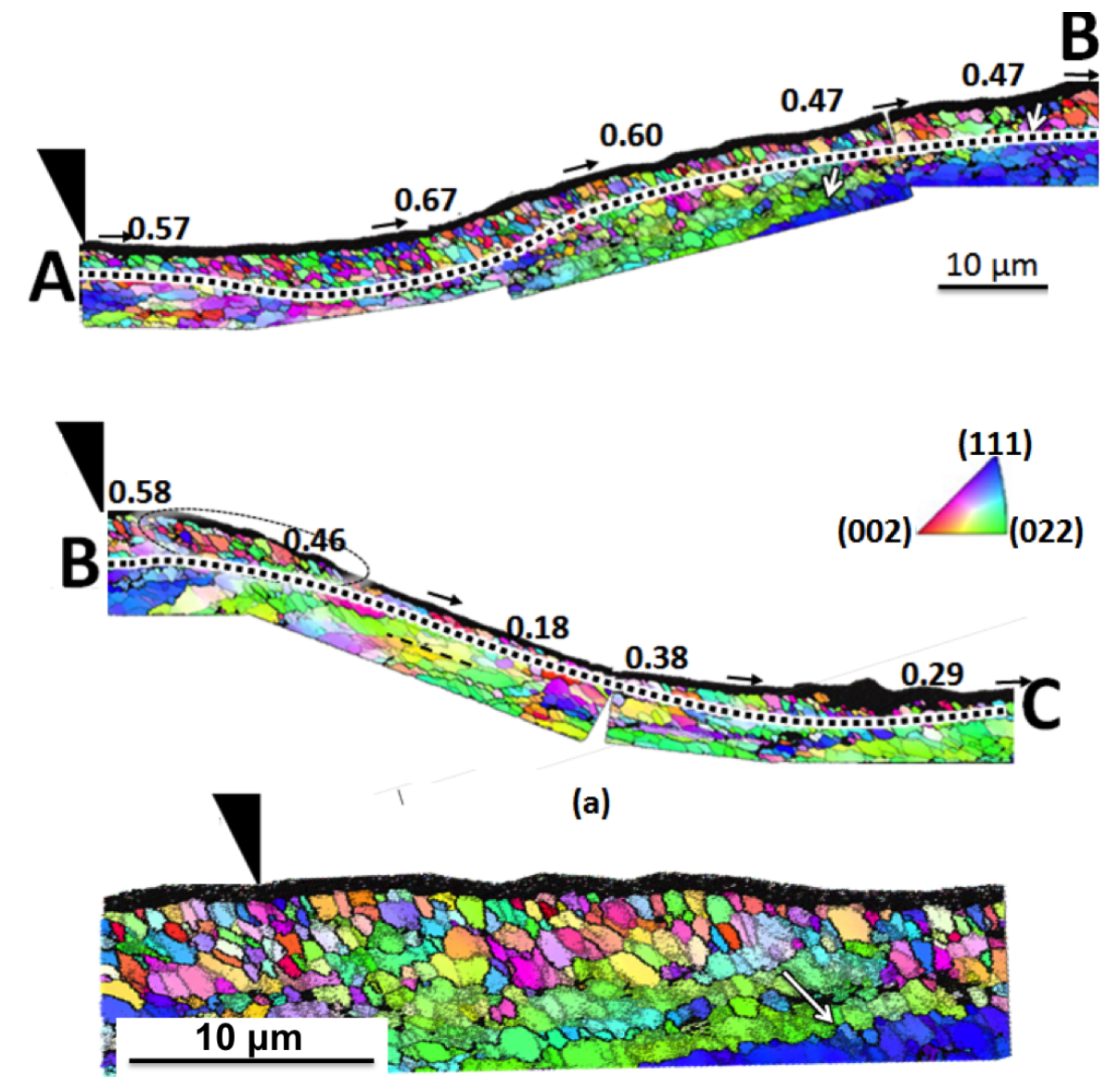

(b)

Figure 3: (a) High resolution OIM of surficial microstructure in transient surface generation. Numbers overlaid above the surface indicate spatial variation in local recrystallization fraction $f_{r x}$. (b) Magnified view of the OIM of surficial microstructure characterized halfway between $\mathrm{AB}$ at $\frac{x}{\lambda}=0.25$. 


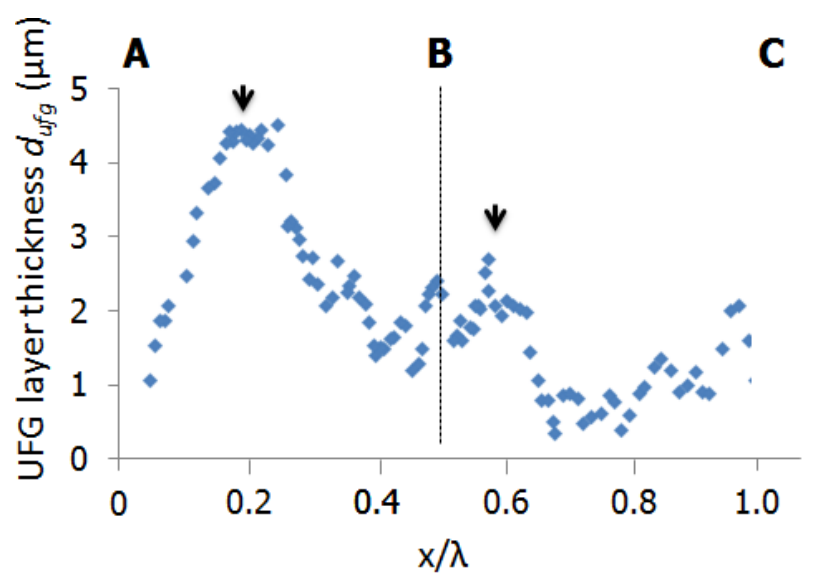

Figure 4: Variation in thickness $d_{u f g}$ of the zone exhibiting UFGs $\delta_{15^{\circ}}<0.5 \mu m$ with respect to normalized position along the surface wavelength $\frac{x}{\lambda}$. 


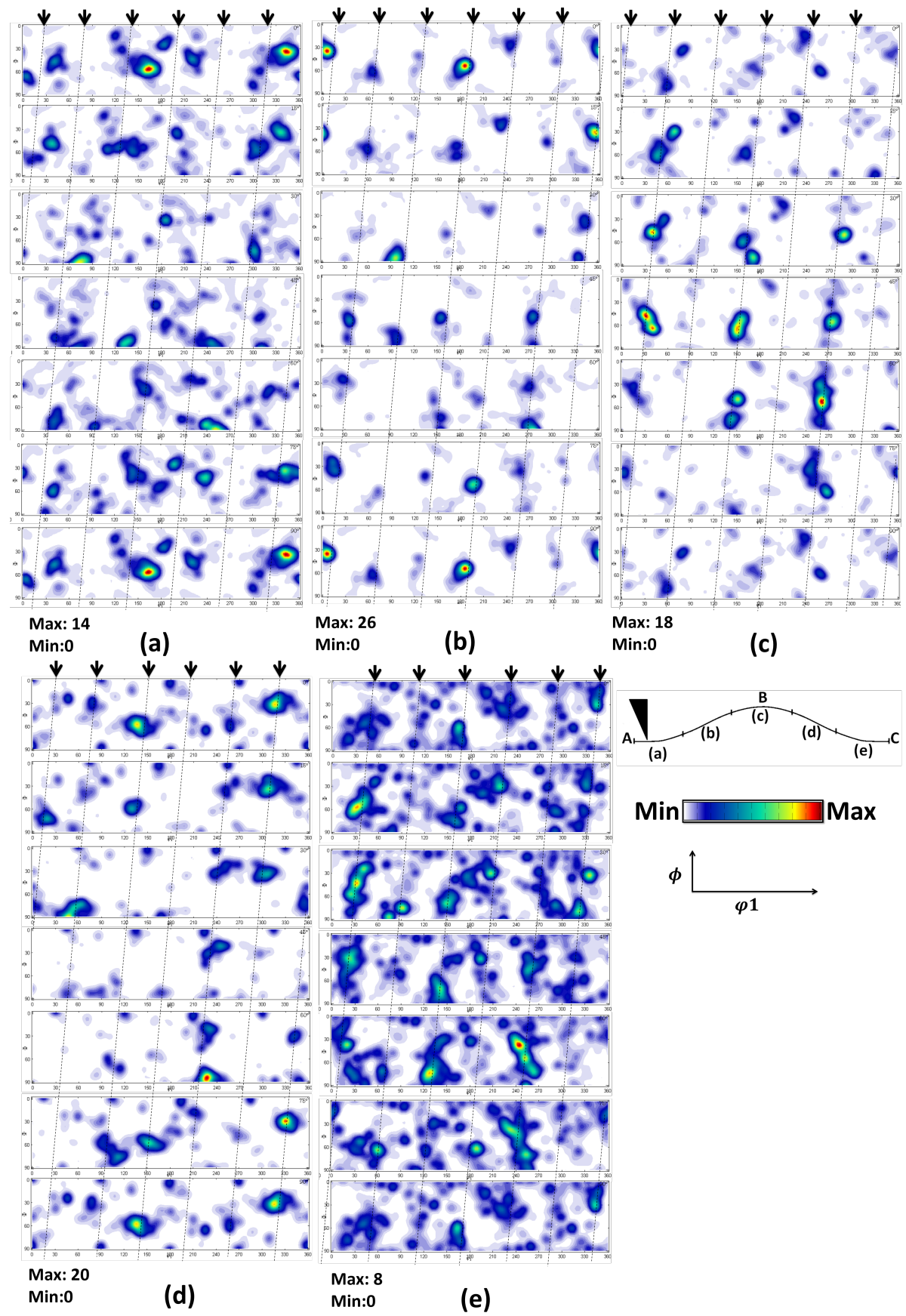

Figure 5: Rotated orientation distribution functions characterized using OIM along locations (a-e) identified in the inset on the bottom right. The rows correspond to $\phi 2=0^{\circ}, 15^{\circ}, 30^{\circ}, 45^{\circ}, 60^{\circ}, 75^{\circ}, 90^{\circ}$ sections. 


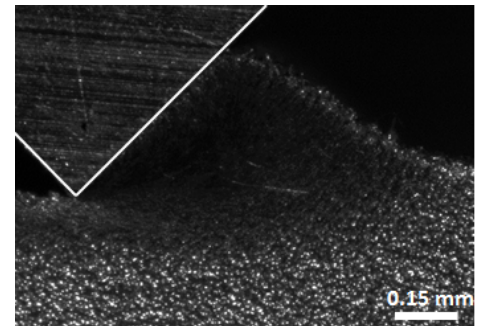

(a)

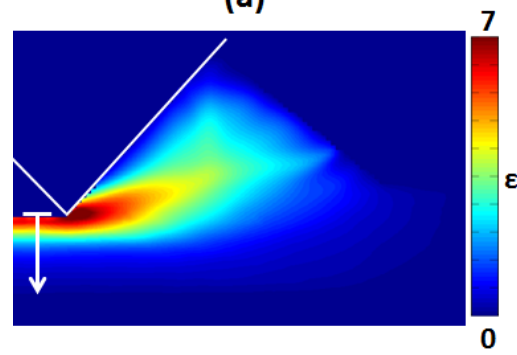

(c)

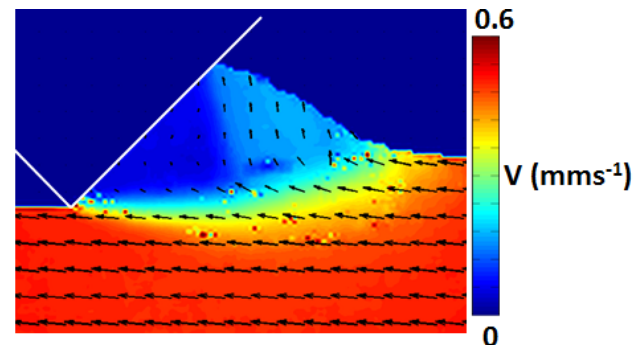

(b)

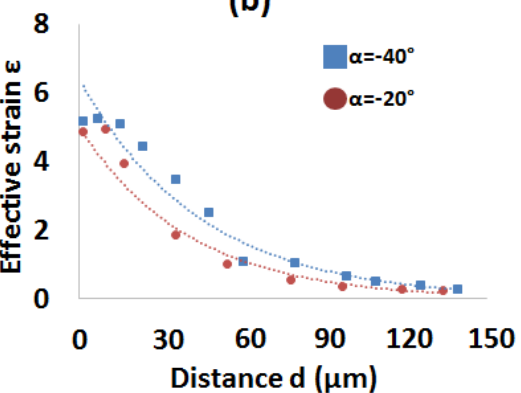

(d)

Figure 6: Characterization of effective strain imposed during surface generation, including (a) representative optical image, (b) measured velocity field and (c) strain field. (d) Exponential variation in strain field with respect to depth $d$ from the surface. Refer arrow in Fig. 6f for depth reference.

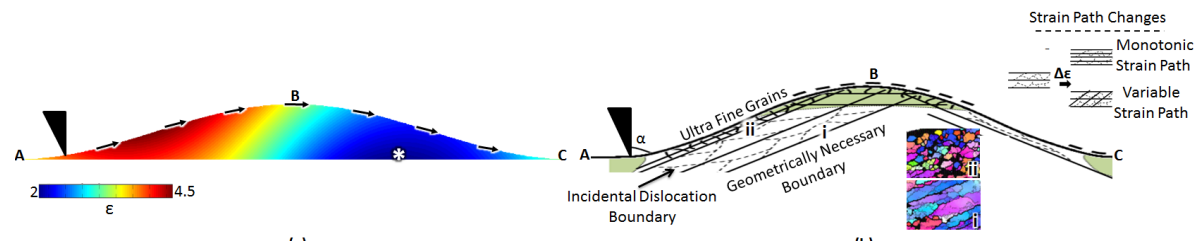

(a)

(b)

Figure 7: (a) Effective strain field imposed on the fabricated topography. (b) Schematic illustrating microstructure evolution in transient surface generation with strain path changes. Inset on right shows effect of strain path changes on accelerated microstructure evolution. These zones are identified using the dashed line. 


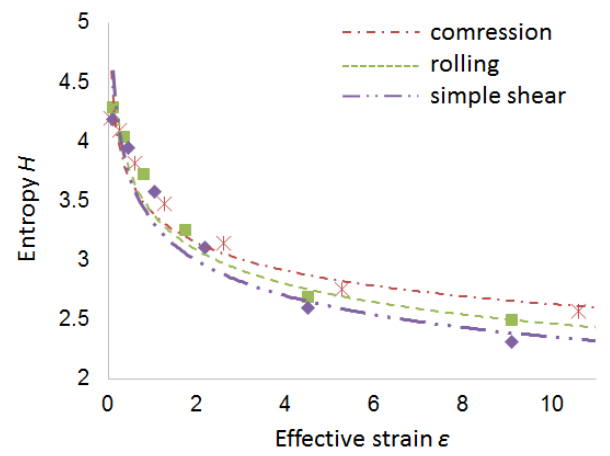

(a)

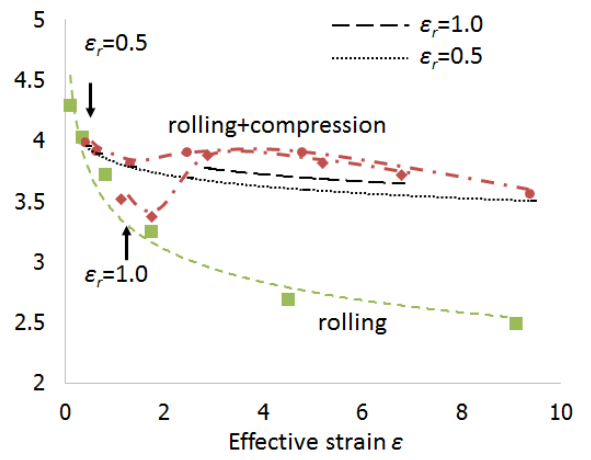

(b)

Figure 8: (a) Variation in entropy $H$ of crystallographic texture with respect to imposed effective strain $\epsilon$ for different monotonic deformation paths. (b) Influence of strain path changes on entropy of ODFs. Strain path changes were imposed at strain levels indicated with black arrows.

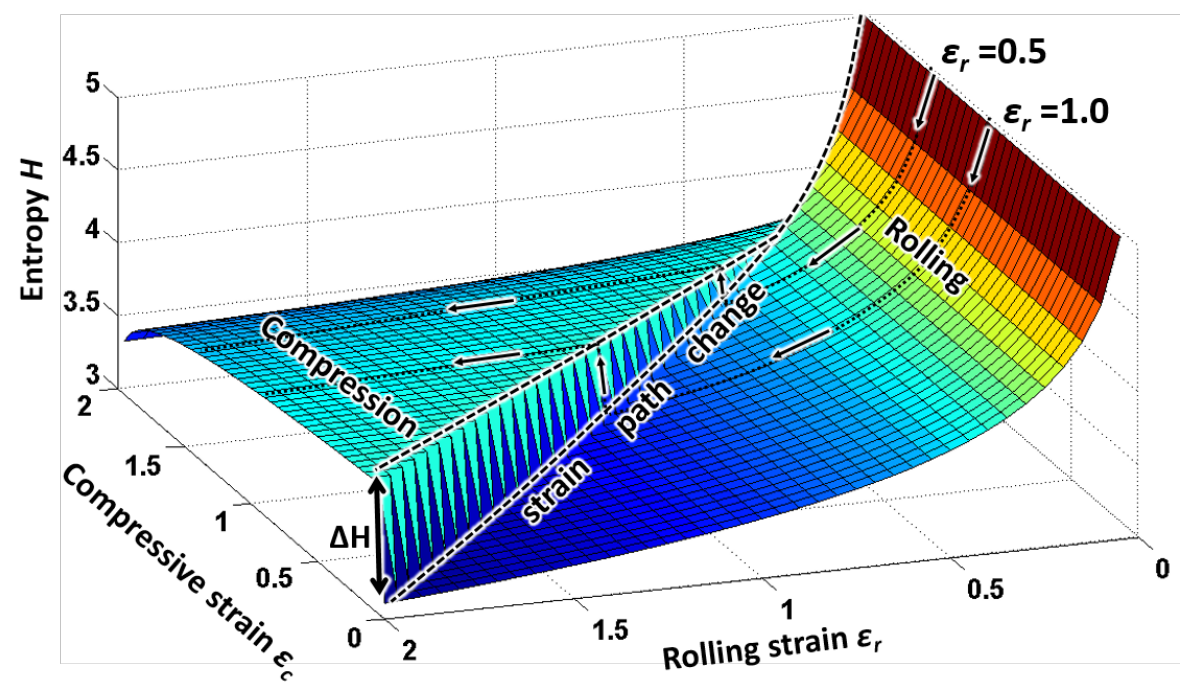

Figure 9: Variation in entropy $H$ and its step changes $\Delta H$ as a consequence of the corresponding step strain path changes. The strain histories demarcated as $\epsilon_{r}=0.5,1$ are also pointed in Fig. 8b. Strain histories should be examined along lines parallel to arrows depicted in Fig. 9. 